\title{
Zentralvorstandssitzung vom 20. Dezember 2012
}

\begin{abstract}
eHealth-Veranstaltungen - Die IG eHealth möchte die verschiedenen Schweizer eHealth-Kongresse in einer neuen Plattform bündeln. Für den Zentralvorstand (ZV) erscheint es sinnvoll, wenn die FMH von Anfang an bei der Vorbereitung und Planung mit dabei ist. Deswegen entscheidet er sich einstimmig für eine Patronatspartnerschaft ohne finanzielle Beteiligung.
\end{abstract}

Übergriffe in ärztlichen Behandlungen - Nach der Diskussion in der Ärztekammer sollen die Formulierungen zunächst innerhalb der Arbeitsgruppe «Sexuelle Übergriffe in ärztlichen Behandlungen» weiterentwickelt werden, für die auch neue Mitglieder vorgeschlagen werden. Der ZV beschliesst, das Thema auf die Traktandenliste einer Sitzung vor der kommenden Ärztekammer am 25. April zu setzen.
Patente auf Dosierungen - Die neue Rechtsprechung lässt Patente auf Dosierungen von Medikamenten zu. Die Vertreter der Pharmaindustrie machten klar, dass sie nicht bereit sind, ein Memorandum of Understanding zu unterzeichnen und anschliessend eine Revision des Patentgesetzes zu akzeptieren. Darauf will der ZV verzichten, sofern ein griffiges Memorandum zustande kommt und eingehalten wird.

Revision Epidemiengesetz - Das revidierte Epidemiengesetz unterscheidet wirksam zwischen Impfzwang und Impfobligatorium, weswegen aus Sicht der FMH eine Unterstützung sinnvoll erscheint. Im Falle einer Referendumsabstimmung beschliesst der $\mathrm{ZV}$, eine Annahme der Vorlage zu empfehlen und für ein gemeinsames Vorgehen Kontakt mit dem Krankenpflegefachverband SBK-ASI aufzunehmen.

\section{Séance du Comité central du 20 décembre 2012}

Rencontres eHealth - La communauté d'intérêt eHealth souhaite réunir les différents congrès suisses dédiés à la cybersanté dans une nouvelle plateforme. Le Comité central (CC) estime judicieux que la FMH soit associée à ce projet dès le départ et a donc décidé à l'unanimité d'opter pour un parrainage sans participation financière.

Abus sexuels lors de l'activité médicale - Lors de la dernière Chambre médicale, il a été décidé que les formulations devront d'abord être revues par le groupe de travail «Abus sexuels lors de l'activité médicale», qui se verra par ailleurs proposer de nouveaux membres. Le CC a décidé d'inscrire ce point à l'ordre du jour de l'une de ses séances précédant la prochaine Chambre médicale du 25 avril.

Brevets sur les dosages - La nouvelle jurisprudence autorise les brevets sur les dosages des médicaments.
Or les représentants de l'industrie pharmaceutique ont indiqué clairement qu'ils n'étaient pas prêts à signer un protocole d'entente ni à accepter ensuite une révision de la loi sur les brevets. Le CC est d'accord de renoncer à cette révision à condition qu'un protocole efficace entre en vigueur et qu'il soit respecté.

Révision de la loi sur les épidémies - Comme la loi révisée sur les épidémies distingue clairement entre «vaccination sous la contrainte» (Impfzwang) et «obligation de vaccination», la FMH estime judicieux de la soutenir. En cas de référendum, le CC a décidé de recommander l'acceptation du projet et de se mettre en rapport avec l'Association suisse des infirmières et des infirmiers (ASI) pour une campagne commune. 